\title{
Molecular Cloning and Characterization of the Vasopressin-regulated Urea Transporter of Rat Kidney Collecting Ducts
}

\author{
Chairat Shayakul, Angela Steel, and Matthias A. Hediger \\ Renal Division, Department of Medicine, Brigham and Women's Hospital and Harvard Medical School, and Department of Biological \\ Chemistry and Molecular Pharmacology, Boston, Massachusetts 02115
}

\begin{abstract}
Absorption of urea in the renal inner medullary collecting duct (IMCD) contributes to hypertonicity in the medullary interstitium which, in turn, provides the osmotic driving force for water reabsorption. This mechanism is regulated by vasopressin via a cAMP-dependent pathway and activation of a specialized urea transporter located in the apical membrane. We report here the cloning of a novel urea transporter, designated UT1, from the rat inner medulla which is functionally and structurally distinct from the previously reported kidney urea transporter UT2. UT1 expressed in Xenopus oocytes mediated passive transport of urea that was inhibited by phloretin and urea analogs but, in contrast to UT2, was strongly stimulated by cAMP agonists. Sequence comparison revealed that the coding region of UT1 cDNA contains the entire 397 amino acid residue coding region of UT2 and an additional 1,596 basepairstretch at the $5^{\prime}$ end. This stretch encodes a novel 532 amino acid residue $\mathrm{NH}_{2}$-terminal domain that has $67 \%$ sequence identity with UT2. Thus, UT1 consists of two internally homologous portions that have most likely arisen by gene duplication. Studies of the rat genomic DNA further indicated that UT1 and UT2 are derived from a single gene by alternative splicing. Based on Northern analysis and in situ hybridization, UT1 is expressed exclusively in the IMCD, particularly in its terminal portion. Taken together, our data show that UT1 corresponds to the previously characterized vasopressin-regulated urea transporter in the apical membrane of the terminal IMCD which plays a critical role in renal water conservation. (J. Clin. Invest. 1996. 98:25802587.) Key words: urea transporter • inner medullary collecting duct • cyclic AMP • vasopressin • alternative splicing
\end{abstract}

\section{Introduction}

The ability of the kidney to concentrate urine during water restriction is central to the survival of mammals in a terrestrial environment (1). This capacity requires accumulation of solutes, mainly urea and $\mathrm{NaCl}$, in the medullary interstitium, to

Address correspondence to Matthias A. Hediger, Renal Division, Brigham and Women's Hospital, 75 Francis Street, Boston, MA 02115. Phone: 617-732-5850; FAX: 617-732-6392; E-mail: mhediger@ bustoff.bwh.harvard.edu

Received for publication 3 June 1996 and accepted in revised form 19 September 1996.

J. Clin. Invest.

(C) The American Society for Clinical Investigation, Inc. 0021-9738/96/12/2580/08 \$2.00

Volume 98, Number 11, December 1996, 2580-2587 generate the hypertonicity and osmotic driving force for water reabsorption (2). Accumulation of urea in the renal medulla mainly results from absorption in the terminal inner medullary collecting duct (IMCD) ${ }^{1}$ and is further enhanced by mechanisms that return urea to the inner medulla after its dissipation toward the outer medulla. These mechanisms involve secretion of urea into thin limbs of loops of Henle in a process called urea recycling and countercurrent exchange between ascending and descending vasa recta (3). The urea permeability varies considerably among different kidney structures with the highest value found in the terminal IMCD, the only segment known to increase its permeability in response to vasopressin $(4,5)$. This unique property of the terminal IMCD allows the delivery of large amounts of urea into the deepest portions of the inner medulla.

The high urea permeability in the terminal IMCD is attributed to a specific phloretin-sensitive, vasopressin regulated urea transporter in the apical membrane $(6,7)$. It is generally believed that the upregulation of the urea permeability in this segment results from the occupation of specific $V_{2}$ vasopressin receptors followed by the activation of adenylate cyclase and intracellular production of cAMP (8). Although the mechanism by which cAMP alters the apical membrane urea permeability is still unclear, the available evidence indicates that urea and water transport in this segment are mediated by physically distinct processes (9).

Previously, our group has isolated the cDNAs encoding mammalian urea transporters from rabbit and rat kidney which are 3.0 and $2.9 \mathrm{~kb}$ in length, respectively, $(10,11)$. Both cDNAs encode a $43-\mathrm{kD}$ protein, called UT2. Expression studies of UT2 in Xenopus oocytes resulted in a large increase in urea transport which was inhibited by phloretin and urea analogs. However, transport of urea in UT2-injected oocytes was not affected by preincubation with cAMP. This result is inconsistent with the previous study by Verkman and colleagues which showed a 4.4-fold increase in the rate of urea uptake in the oocytes injected with mRNA from the renal medulla after exposure to cAMP agonists (12). This raises the possibility that an additional urea transporter which is vasopressin regulated exists in the inner medulla.

Based on high stringency Northern analysis using fulllength UT2 cDNA as a probe, we have described an additional $4.0-\mathrm{kb}$ transcript localized both in rabbit and rat inner medulla $(10,11)$. To fully interpret the roles of urea transporters in urinary concentration, the present study was designed to identify and characterize this $4.0-\mathrm{kb}$ transcript in the rat inner medulla. Our results demonstrate that this transcript is different from UT2 and that it encodes a unique vasopressin-regulated urea

1. Abbreviations used in this paper: IMCD, inner medullary collecting ducts; nt, nucleotide. 
transporter localized in the IMCD, hereafter referred to as UT1. We also present evidence that UT1 and UT2 are produced by alternative splicing and propose possible mechanisms for splice site selection.

\section{Methods}

Clone isolation. A rat kidney inner medullary cDNA library was constructed from gel-purified cDNA $(>3.5 \mathrm{~kb})$ in bacteriophage $\lambda \mathrm{gt} 10$. The cDNA contained NotI and SalI restriction sites on either end to facilitate further subcloning. Approximately 500,000 clones were screened at high stringency using the full-length rat UT2 cDNA as a probe. A positive clone containing a 4.0-kilobase insert (UT1) was identified and subcloned into pBluescriptIISK ${ }^{-}$. Both strands were sequenced by the dideoxy chain termination method with $\mathrm{Se}$ quenase version 2.0 (USB Biologicals, Cleveland, $\mathrm{OH}$ ).

In vitro transcription and translation. cRNA was synthesized in vitro from the HindIII cut UT1 clone using T3 RNA polymerase and microinjected into collagenase-treated and manually defolliculated Xenopus oocytes. The expression of the urea transporter UT1 was studied by measuring the uptake of $\left[{ }^{14} \mathrm{C}\right]$ urea as previously described (10). For inhibition experiments, $\left[{ }^{14} \mathrm{C}\right]$ urea uptake was measured after a 30 -min preincubation with $0.7 \mathrm{mM}$ phloretin, $150 \mathrm{mM}$ thiourea, $150 \mathrm{mM}$ dimethylurea, or $1 \mathrm{mM}$ p-chloromercuribenzenesulfonate (pCMBS).

The effect of cAMP on UT1 expression in the oocytes was investigated by using the membrane-permeable analog of cAMP, dibutyryl cAMP, in conjunction with forskolin $(50 \mathrm{mM})$ which activates cAMP production by adenylate cyclase, and 3-isobutyl-1-methylxanthine (IBMX, $0.5 \mathrm{mM}$ ) which blocks cAMP degradation by phosphodiesterases. A mixture of these cAMP agonists was present during the indicated preincubation period as well as during the urea uptake measurements.

cRNA was also translated in vitro using rabbit reticulocyte lysates in the presence and absence of canine pancreatic microsomes (Promega Corp., Madison, WI), as previously described (13). The translation products were analyzed by $10 \%$ SDS-PAGE.

Northern analysis. The cDNA probe used for Northern analysis contained the first 652 nucleotides at the $5^{\prime}$ terminus of UT1 cDNA. Sequence analysis showed that this region is different from UT2. For Northern analysis, poly(A) ${ }^{+}$RNA ( $3 \mu \mathrm{g} /$ lane) or total RNA $(10 \mu \mathrm{g} /$ lane) were separated by electrophoresis in a formaldehyde/agarose gel, and the RNA was capillary blotted onto a nitrocellulose filter. The filter was hybridized at $42^{\circ} \mathrm{C}$ in $50 \%$ formamide and washed in buffer containing $0.1 \times \mathrm{SSC}, 0.1 \%$ SDS at $42^{\circ} \mathrm{C}$ (low stringency) or at $65^{\circ} \mathrm{C}$ (high stringency). The same filter was also used for hybridization with cDNA probe synthesized from the first 708 nucleotides of $\mathrm{UT} 2$, a region which is distinct from UT1.

In situ hybridization. These experiments were performed as previously described (14) using 4\% paraformaldehyde-fixed parasaggital tissue sections of rat kidney (5- $\mu \mathrm{m}$ thickness). The cRNA probes used were synthesized either from full-length UT2 cDNA or from the unique $5^{\prime}$ regions of UT1 or UT2, respectively. To synthesize fulllength UT2 probes, ${ }^{35} \mathrm{~S}$-labeled sense or antisense cRNAs were synthesized from UT2 cDNA in pSPORT1 after linearization with NotI or SalI, using T7 or SP6 RNA polymerase, respectively. To synthesize subtype specific probes of UT1, sense or antisense cRNAs were synthesized from a subclone containing the first 652 nucleotides (nt) in pBluescript II SK ${ }^{-}$after linearization with HindIII or NotI, using T3 or T7 RNA polymerase, respectively. To synthesize subtype specific probe of UT2, the sense or antisense cRNAs were synthesized from the subclone of the first 708 nucleotides in pBluescript II SK ${ }^{-}$after linearization with BamHI or HindIII, using T7 or T3 RNA polymerase, respectively. The cRNAs were hydrolyzed for $13 \mathrm{~min}$ to form probes of $\sim 100$ nucleotides in length. After hybridization at $50^{\circ} \mathrm{C}$ overnight, the dried tissue sections were exposed to Biomax MR film (Eastman Kodak Co., Rochester, NY) for $2 \mathrm{~d}$. Tissue slides were coated with Kodak NTB2 emulsion and stored for $7 \mathrm{~d}$ before developing and counterstaining with hematoxylin and eosin.

Studies of rat genomic DNA. Sequence analysis of UT1 cDNA revealed identity to UT2 in the $3^{\prime}$ portion of the coding region and in the $3^{\prime}$ untranslated region but not in the $5^{\prime}$ regions (see Discussion). This finding suggests that both mRNAs are transcribed from a single gene by using a common $3^{\prime}$ exon group (exon group 3 ) but different $5^{\prime}$ exon groups (exon group 1 or exon group 2 for UT1 or UT2, respectively). To confirm this hypothesis, we performed PCR amplification of rat genomic DNA to determine the alignment of each exon group and the introns. The location of PCR primers are indicated in Fig. 6. The following two pairs of sense and antisense oligonucleotide primers were used: pair I-1: 5'-AGCCTATGCACTGCCTGTTGG-3' [bases 159-179 of UT2] and 5'-CTGATGTCTGCTGTC TAGGCC-3' [bases 42-22 of UT1]; pair I-2: 5'-CTGGTGGCTCTGTTTATCTCC-3' [bases 1316-1336 of UT1] and 5'-CCTGCATCTTCCAGCCTTTCC-3' [bases 577-557 of UT2]. These primer pairs were designed to determine whether the exon group 1 is located downstream or upstream of exon group 2. To confirm the distance of the exon groups 1 and 2, respectively, from exon group 3, the following two primer pairs were used: pair I-3: 5' -CTGGTGGCTCTGTTTATCTCC-3' [bases 1316-1336 of UT1] and 5'-ATATGGAACACGCTCTTACGC-3' [bases 800-780 of UT2]; pair I-4: 5'-AGCCTATGCACTGCCTGTTGG-3' [bases 159-179 of UT2] and 5'TCCGTGTGACTGTTCTCC-3' [bases 733-716 of UT2]. The PCR reactions were carried out using $1 \mu \mathrm{g}$ of rat genomic DNA, $30 \mathrm{nM}$ of each primer, $200 \mu \mathrm{M}$ deoxynucleoside triphosphate, and $4.0 \mathrm{U}$ of rTth DNA polymerase, XL (Perkin Elmer Corp., Foster City, CA) in a final volume of $100 \mu \mathrm{l}$ following the manufacturer's recommendation. For control reactions, total rat kidney cDNA was used instead of rat genomic DNA. Amplified products were separated by electrophoresis in a $0.8 \%$ agarose gel and transferred onto a nylon membrane for Southern analysis.

\section{Results}

cDNA cloning and functional characterization of UT1. By screening a rat kidney inner medullary cDNA library with the full-length rat UT2 cDNA as a probe, a 4.0-kb cDNA encoding a novel urea transporter (UT1) was isolated. When expressed in Xenopus oocytes, UT1 induced the uptake of $\left[{ }^{14} \mathrm{C}\right]$ urea $\sim 15$ - to 20 -fold above control values (90 s uptake of $1 \mathrm{mM}$ urea, Fig. $1 a$ ) but the uptake of small solutes of the similar size such as $\left[{ }^{14} \mathrm{C}\right]$ glycerol was not affected (60 min uptake of $1 \mathrm{mM}$ glycerol, data not shown). The increase in urea uptake was almost completely inhibited by $0.7 \mathrm{mM}$ phloretin, $150 \mathrm{mM}$ thiourea, and $150 \mathrm{mM}$ dimethylurea (Fig. 1 a). However, preincubation with $1 \mathrm{mM}$ pCMBS for $30 \mathrm{~min}$ did not affect the urea transport rate of UT1 expressing oocytes (data not shown). The time course of urea uptake in oocytes expressing UT1 is shown in Fig. $1 b$. The uptake of $\left[{ }^{14} \mathrm{C}\right]$ urea $(1 \mathrm{mM})$ reached a maximum of about 230 pmol per oocyte in $60 \mathrm{~min}$.

Whereas preincubation with a mixture of cAMP agonists had no effect on urea uptake in oocytes expressing UT2 or in water-injected oocytes, cAMP induction significantly increased urea uptake in the oocytes expressing UT1. Incubation with forskolin $(50 \mu \mathrm{M})$, IBMX $(0.5 \mathrm{mM})$, and $0.1 \mathrm{mM}$ dibutyryl cAMP for 30 min increased urea uptake 1.9-fold over the controls and 3.6-fold when the dibutyryl cAMP concentration was increased to $0.5 \mathrm{mM}$ (Fig. 1c). The stimulatory effect of cAMP on the uptake of urea was dependent on the preincubation time. The 90-s urea uptakes of UT1-expressing oocytes reached a maximum of about fivefold over control after a 60min preincubation (Fig. $1 d$ ). 

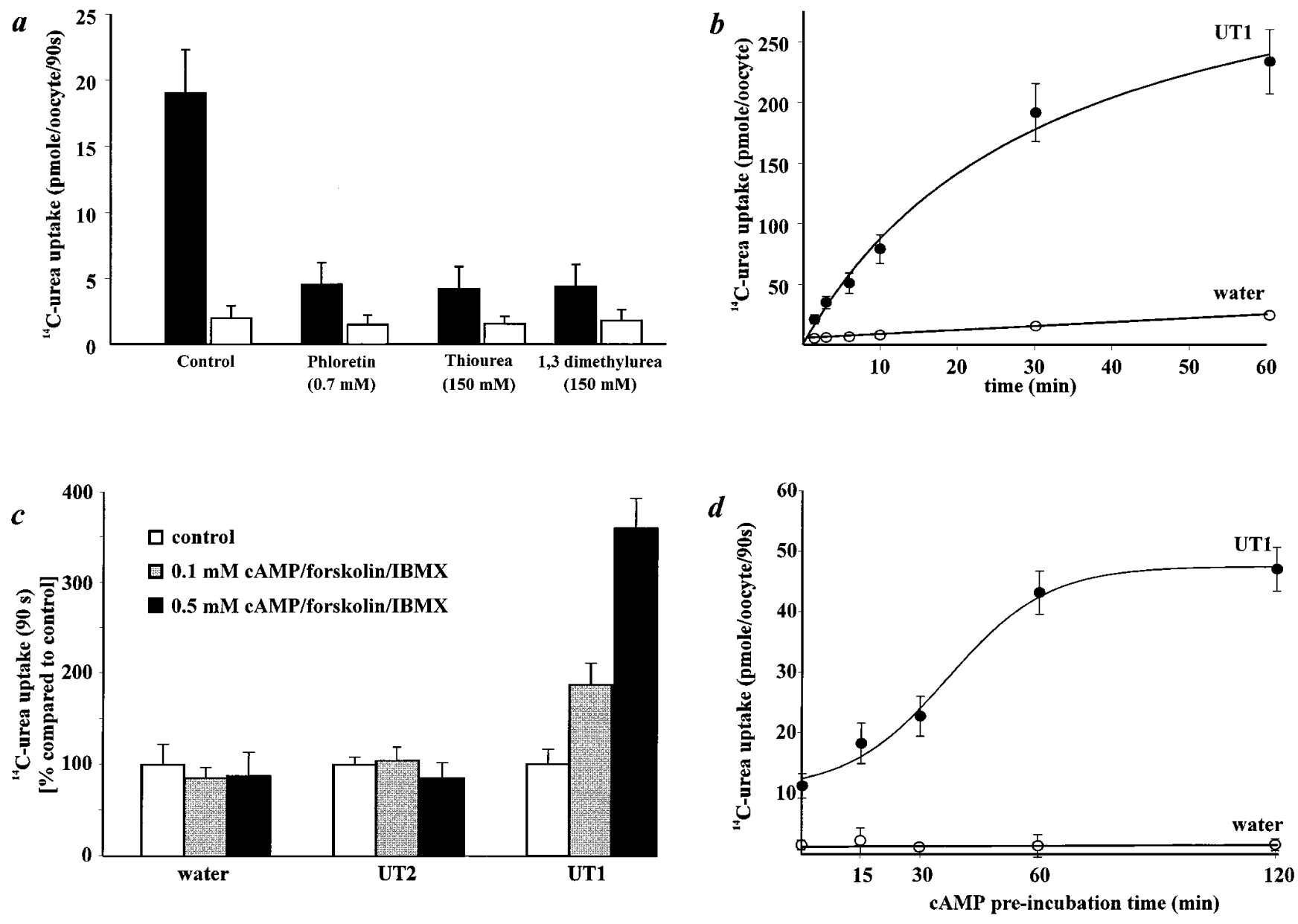

Figure 1. Expression of UT1 in Xenopus oocytes. (a) The uptake of $\left[{ }^{14} \mathrm{C}\right]$ urea $(1 \mathrm{mM})$ by UT1 cRNA-injected oocytes $(1 \mu \mathrm{g} / \mu \mathrm{cRNA}$ injected/ oocyte, filled bars) was compared with water injected oocytes (open bars) during a 90-s incubation. Bars represent mean \pm SD $(n=6-8$ oocytes). $\left[{ }^{14} \mathrm{C}\right]$ urea uptake was effectively inhibited by phloretin $(0.7 \mathrm{mM})$, thiourea $(150 \mathrm{mM})$, and dimethylurea $(150 \mathrm{mM})$. $(\mathrm{b})$ Time course of urea uptake $(1 \mathrm{mM})$ in the UT1-expressing oocytes. (c) Effect of cAMP agonists on the urea uptake $(1 \mathrm{mM}, 90 \mathrm{~s})$ of oocytes injected with water, UT2 cRNA or UT1 cRNA. Oocytes were preincubated with 0.1 or $0.5 \mathrm{mM}$ of dibutyryl cAMP, in addition to forskolin and IBMX, for $60 \mathrm{~min}$. These agents were also present during the uptake measurement. $(d)$ Time course of cAMP-stimulated urea uptake in UT1-expressing oocytes. Oocytes expressing UT1 were preincubated with $0.5 \mathrm{mM}$ dibutyryl cAMP, forskolin, and IBMX during the indicated time period and the uptake of $1 \mathrm{mM}$ $\left[{ }^{14} \mathrm{C}\right]$ urea was subsequently studied during $90 \mathrm{~s}$ and in the presence of these agents. Each value is mean $\pm \mathrm{SD}$ for $6-8$ oocytes from two to three separate studies.

Analysis of the UT1 amino acid sequence. UT1 cDNA has an open reading frame from nucleotides 377 to 3163 that encodes a 929 amino acid residue protein with a predicted molecular weight of $102 \mathrm{kD}$. Although there is no characteristic Kozak consensus initiation sequences at nucleotide 377 , the GCG program 'TESTCODE' identified this as a potential initiation site. Accordingly, in vitro translation of UT1 cRNA using rabbit reticulocyte lysates yielded a protein with an apparent $M_{\mathrm{r}}$ of $76 \mathrm{kD}$ in the absence of, and $86 \mathrm{kD}$ in the presence of canine pancreatic microsomes. These sizes are roughly twice as large as those obtained for UT2 cRNA (40 and $45 \mathrm{kD}$, respectively). The shift in $M_{\mathrm{r}}$ of UT1 in the presence of microsomes was reversed by deglycosylation treatment with endoglycosidase-H (not shown). Two potential N-glycosylation sites were identified at Asn 279 and 742 (Fig. $2 a$ ).

Hydropathy analysis of the deduced amino acid sequence using a window of 21 shows that UT1 consists of two similar halves, each of which is composed of two extended hydropho- bic membrane spanning stretches as previously identified in UT2 (Fig. 2). Sequence analysis using the GCG sequence analysis software (Promega Corp.) revealed internal homology between these two halves with $67.4 \%$ identity between the amino-terminal half (UT1-A) and the carboxy-terminal half (UT1-B) (Fig. $3 a$ ). In addition, each half of UT1 can be further subdivided into two homologous hydrophobic domains (UT1-A1/ UT1-A2 and UT1-B1/ UT1-B2) with percentage identities ranging from 21.7 to $25.9 \%$ (UT1-A1:UT1-A2 = 25.9\%, UT1-A1:UT1-B2 = 21.7\%, UT1-A2:UT1-B1 = 23.2\%, and UT1-B1:UT1-B2 $=24.7 \%$ ) (Figs. $2 b$ and $3 b$ ).

UT1 $m R N A$ expression and tissue distribution. Northern analysis using subtype specific probes corresponding to the first $652 \mathrm{nt}$ of UT1 revealed two transcripts of 4.0 and $2.2 \mathrm{~kb}$ localized specifically in the inner medulla, particularly in the innermost part. No signal was detected in either the remaining parts of the kidney or in other organs including colon, liver, lung, brain, and bone marrow (Fig. 4 a). In contrast, using 
a
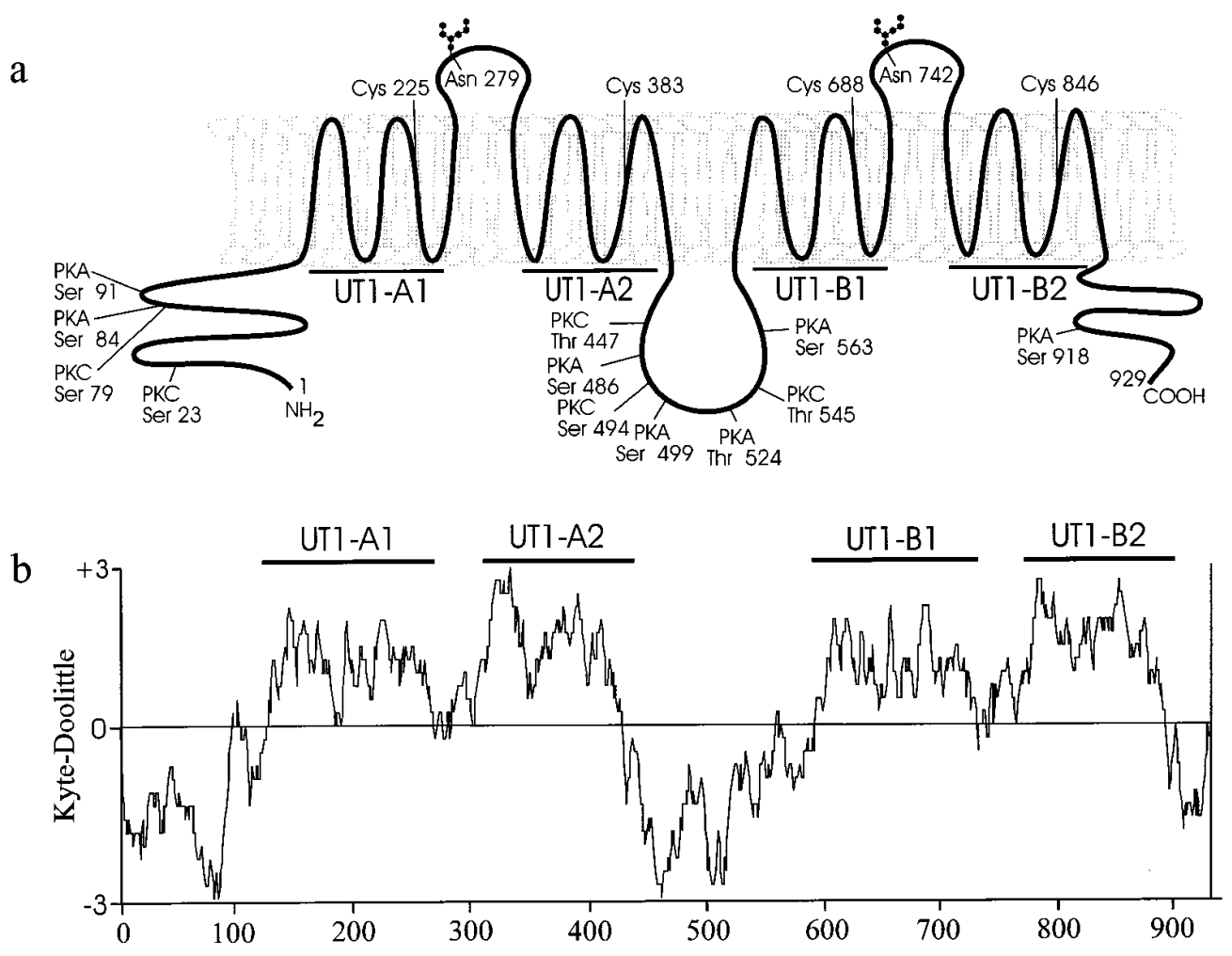

Figure 2. Hypothetical structural model and hydropathy analysis of UT1. (a) Schematic representation of the membrane topology of UT1. Regions UT1-A1, UT1-A2, UT1$\mathrm{B} 1$, and UT1-B2 represent the four internal hydrophobic repeats. Putative PKA and PKC phosphorylation sites and two potential $N$-glycosylation sites are indicated. The conserved extracellular cysteine residues are also marked (see asterisk Fig. $3 b$ ). (b) Kyte-Doolittle hydropathy analysis of the UT1 amino acid sequence using a window of 21. The four extended hydrophobic repeats are highlighted.
UT2-specific probes, the expression of the 2.9-kb transcript was found only in the kidney outer medulla and outer half of inner medulla (Fig. $4 b$ ).

In situ hybridization was used to determine the exact distribution of UT1 and UT2 mRNA in rat kidney. When using the full-length antisense UT2 cRNA as a probe, signals were detected both in the inner stripe of the outer medulla and in the inner medulla. However, when using subtype specific probes for UT2 and UT1, UT2 signals were detected mainly in the inner stripe of the outer medulla and the upper part of the inner medulla, whereas UT1 signals were found only in the IMCD, particularly in its terminal part (Fig. 5).

Genomic organization of the urea transporter gene. Sequence comparison of full-length UT1 cDNA showed complete identity with UT2 in its $3^{\prime}$ terminus but not in its $5^{\prime}$ terminus. The first 1751 nucleotides at the $5^{\prime}$ terminus of UT1 are unrelated to the $5^{\prime}$ untranslated 707 nucleotides of UT2. This intriguing sequence relationship between UT1 and UT2 suggests that both mRNAs are derived from a single gene by utilization of alternative splice sites at the $5^{\prime}$ region and that there must be at least three exon groups involved in this process (Fig. $6 a$ ): The UT1 5' exon group (1751 nt, exon group 1), the UT2 $5^{\prime}$ exon group (707 nt, exon group 2), and the common $3^{\prime}$ exon group (2228 nt, exon group 3). To validate this organization, we used the oligonucleotide primer pairs I-1, I-2, I-3, and I-4 (see Fig. $6 a$ ) which were designed to determine the order of the exon groups and the size of the intervening genomic sequences. Whereas no significant band was detected by PCR using primer pair I-1, amplified products of $\sim 7.0 \mathrm{~kb}$ were identified using primer pair I-2 (Fig. $6 \mathrm{~b}$ ). We therefore conclude that exon group 1 is located $\sim 7.0 \mathrm{~kb}$ upstream of exon group 2 which itself precedes exon group 3 . PCR amplification of rat genomic DNA using primer pair I-4 produced a product equivalent in size to that obtained from total rat kidney cDNA (Fig. $6 b$ ). This indicates that there is no intron between exon groups 2 and 3 . Sequence analysis of the boundaries between exon groups 2 and 3 revealed that there is a $3^{\prime}$ consensus acceptor site (CAG) (nt 705-707), in agreement with a functional splicing site. Taken together, these results indicate that UT1 and UT2 are produced by alternative splicing of a single gene by utilization of different exon groups at the $5^{\prime}$ terminus.

\section{Discussion}

In addition to the well-known effect of vasopressin to increase water permeability in the collecting duct, vasopressin also plays an important role in regulating urea transport. Increased urea transport in response to vasopressin occurs only in the terminal IMCD, the primary site responsible for accumulation of urea in the inner medulla. Consequently, urea transport across the terminal IMCD and its regulation by vasopressin are crucial components of the urinary concentrating mechanism. The high urea permeability in this segment is attributable to specialized phloretin-sensitive urea transporters in the apical and basolateral cell membranes. However, it has been shown that the rate-limiting step for vasopressin-stimulated transepithelial urea transport is the apical membrane (15) and that cAMP is the second messenger that mediates this response (8).

A previous study showed that preincubation with cAMP increased the urea uptake in oocytes injected with mRNA from kidney inner medulla, a characteristic that was not observed for the previously reported urea transporter UT2 (10). This finding suggested that a urea transporter other than UT2 is expressed in the terminal IMCD. By screening a rat kidney inner medullary cDNA library with the rat UT2 probe, we 
Figure 3. Alignment of homologous repeats of UT1. (a) Amino acid sequence alignment of the first $\mathrm{NH}_{2}$-terminal half (UT1-A from residues 1-532) with the second $\mathrm{COOH}$-terminal half (UT1-B from residues 533-929) using the Bestfit program. Gaps required for optimal alignment are shown as dots and identical amino acids are boxed. (b) Sequence comparison of the four homologous internal repeats with the conserved amino acids (at least three out of four) marked in bold and underlined. The Asn-Pro motif, common in aquaporins, is highlighted by two crosses and the asterisk identifies the conserved extracellular cysteine residue. The bracket indicates a signature sequence of the UT family. The nucleotide sequence of UT1 has been deposited in the GenBank under accession No. U77971.

have isolated a $4.0-\mathrm{kb}$ cDNA clone that encodes a novel urea transporter, UT1. Northern analysis and in situ hybridization with specific probes generated from the $5^{\prime}$ region of UT1 cDNA revealed that expression of UT1 is restricted to the IMCD, in particular to its terminal part, whereas UT2 is expressed mainly in the inner stripe of outer medulla and in the upper part of the inner medulla. This finding, in conjunction with the unique functional transport characteristics, strongly indicates that UT1 is the phloretin-sensitive, vasopressin-regulated urea transporter in the IMCD of rat kidney.

Studies using isolated perfused rat terminal IMCD demonstrated two phases of vasopressin stimulation and withdrawal: an initial phase in which urea transport increases rapidly within 5-10 min and a late phase with a slower increase that is complete within 30-60 min $(8,9,16)$. This effect was mimicked by incubation with $1 \mathrm{mM} 8$-Br-cAMP (16). Molecular identification of UT1 enabled us to directly investigate the cellular mechanism involved in vasopressin regulation of the terminal IMCD urea transporter. In agreement with isolated perfused tubule studies, pre-incubation of oocytes expressing UT1 with cAMP agonists for 60 min produced a fivefold stimulation in the urea uptake. The initial rapid phase of stimulation, however, was not detected in the oocytes expression system. The available evidence suggests that vasopressin enhances the urea permeability in the terminal IMCD by increasing the number of functional transporters either by a membrane shuttling mechanism and/or by in situ activation of the transporter ( 6 , 17). At present, there is no evidence available that the endocytosis/exocytosis process is functioning properly in Xenopus oocytes. UT1 has 12 potential phosphorylation sites, 7 for protein kinase A (PKA) and 5 for protein kinase C (PKC) (Fig. 2). Taken together, it is likely that direct phosphorylation contributes, at least in the late second phase, to the up-regulation of UT1 in response to vasopressin, as suggested by previous studies $(10,12)$.

From a structural perspective, UT1 consists of two internally homologous repeats, a finding which is comparable with those members of the aquaporin family. These channels consist of two tandem repeats that are predicted to be orientated $180^{\circ}$ to each other, thereby forming a water pore. The motif Asn-Pro-Ala of aquaporins is predicted to be part of the pore forming region (18). It is of interest to note that, although there is no significant homology between water channels and urea transporters, the urea transporter repeats UT1-A1, UT1A2, UT1-B1, and UT1-B2 contain a similar motif: Asn-ProLeu/Trp (see Fig. 3). This motif could conceivably form part of the urea translocation pathway.

Concerning the similarities in protein structure of each half 


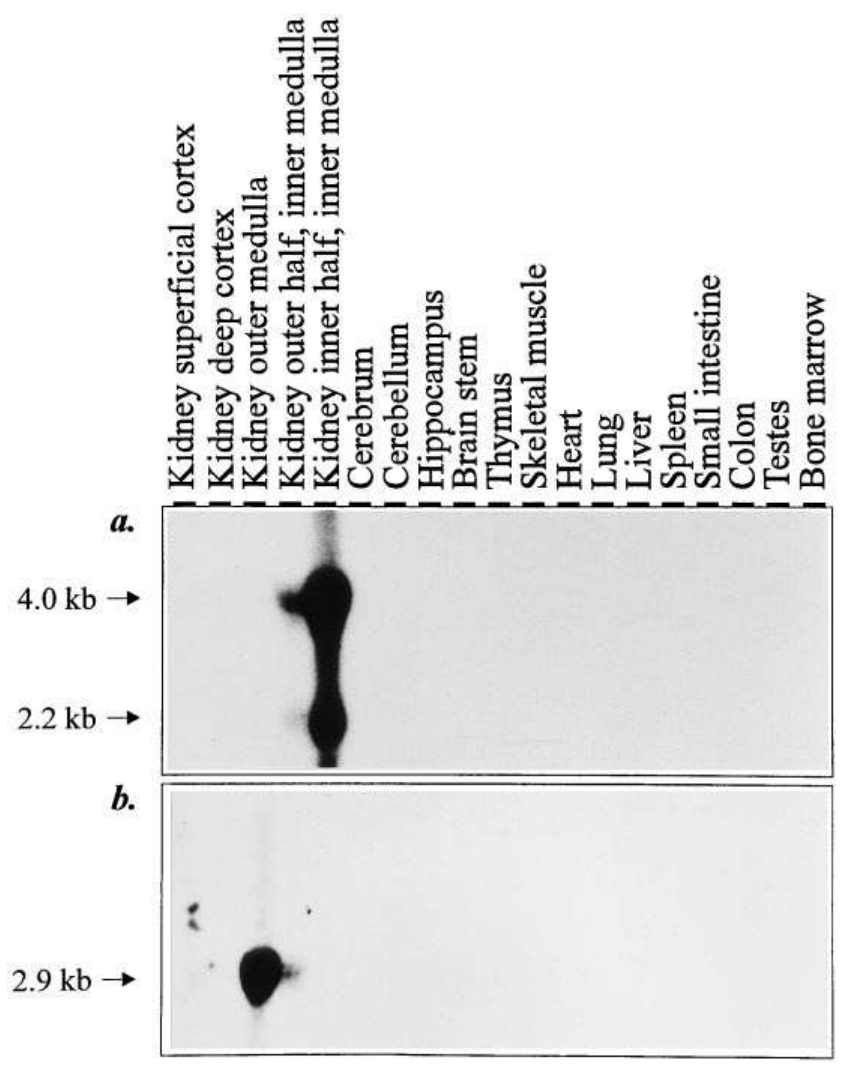

Figure 4. Tissue distribution of UT1 $(a)$ and UT2 $(b)$ in rat by high stringency Northern analysis. Total RNA $(10 \mu \mathrm{g})$ from the outer and inner halves of the kidney inner medulla and bone marrow, or poly $(\mathrm{A})^{+}$RNA ( $3 \mu \mathrm{g}$ ) from other rat tissues were electrophoresed in a $1 \%$ formaldehyde agarose gel and transferred onto a nylon membrane. (a) The filter was probed with a UT1 specific probe, constructed from the first $652 \mathrm{nt}$ of UT1 cDNA, yielding strong 4.0- and $2.2-\mathrm{kb}$ signals in the inner half of the inner medulla and weaker signals in the outer half of the inner medulla. (b) The same filter was probed with a UT2-specific probe generated from the first $708 \mathrm{nt}$ of UT2 cDNA. A 2.9-kb transcript was strongly expressed in the outer medulla and weakly in the outer half of the inner medulla. Filters were hybridized at $42^{\circ} \mathrm{C}$, washed at high stringency $\left(65^{\circ} \mathrm{C}\right)$ in buffer containing $0.1 \times \mathrm{SSC} / 0.1 \%$ SDS, and autoradiographed for $72 \mathrm{~h}$ at $-80^{\circ} \mathrm{C}$.

of UT1 and UT2, it is likely that both urea transporters evolved from the same ancestral gene by duplication and divergence. The unique hydrophobic structure of these proteins is present in all members of the urea transporter protein family currently identified, including the human erythrocyte urea transporter HUT11 which is also expressed in the kidney (19). It seems reasonable to predict that these isoforms evolved to provide a more refined mechanism to regulate water balance in different physiological states of land vertebrates.

The present study on the rat genomic DNA indicates that UT1 and UT2 are alternative splice products that are derived from a single gene by differential utilization of alternative $5^{\prime}$ exon groups. Since the localization and regulation of UT1 and UT2 expression are spatially distinct (see above and reference 11), the selection of splice sites must be regulated in a tissuespecific manner either by differentially expressed trans-acting factors and/or by two distinct promoters (cis factors) located
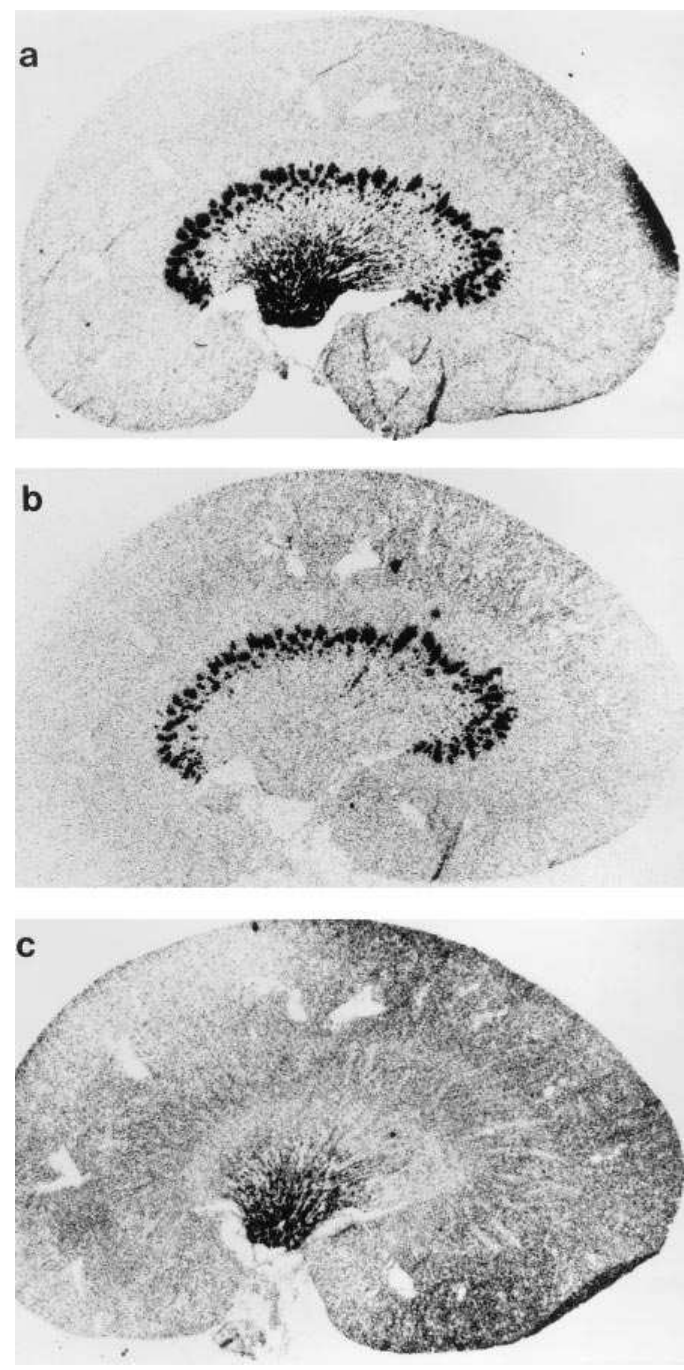

Figure 5. Localization of UT1 and UT2 mRNA expression by in situ hybridization of rat kidney. (a) Parasagittal cryosections of rat kidney were probed with ${ }^{35}$ S-labeled antisense UT2 cRNA synthesized from full-length UT2 cDNA and hydrolyzed to generate probes of $\sim 100$ nt. Because the full-length UT2 probe hybridizes to both UT1 and UT2 mRNA, the signals are evident both in the inner medulla and inner stripe of the outer medulla. (b) The antisense UT2 specific probe, constructed from the first $708 \mathrm{nt}$ of UT2, produced specific signals that were found mostly in the inner stripe of the outer medulla. Autoradiographs show a representative result from one of three kidneys analyzed. $(c)$ Hybridization of antisense UT1 specific cRNA probe synthesized from the first $652 \mathrm{nt}$ of UT1 gave specific signals in the inner medullary collecting duct.

upstream of each $5^{\prime}$ exon group. The latter mechanism appears to be more likely considering the orientation of the two $5^{\prime}$ exon groups within the genome. It is of interest to note that vasopressin has different long term effects on the level of expression of UT1 and UT2 mRNA in rat (Shayakul, C., C.P. Smith, H.S. Mackenzie, W.S. Lee, and M.A. Hediger, manuscript in preparation). cAMP has been shown to regulate the transcriptional rate of specific genes by phosphorylation of transcription factors which then bind to cAMP responsive elements located in the promoter regions (20). It is therefore possible that the promoter region of the rat urea transporter gene contains a 


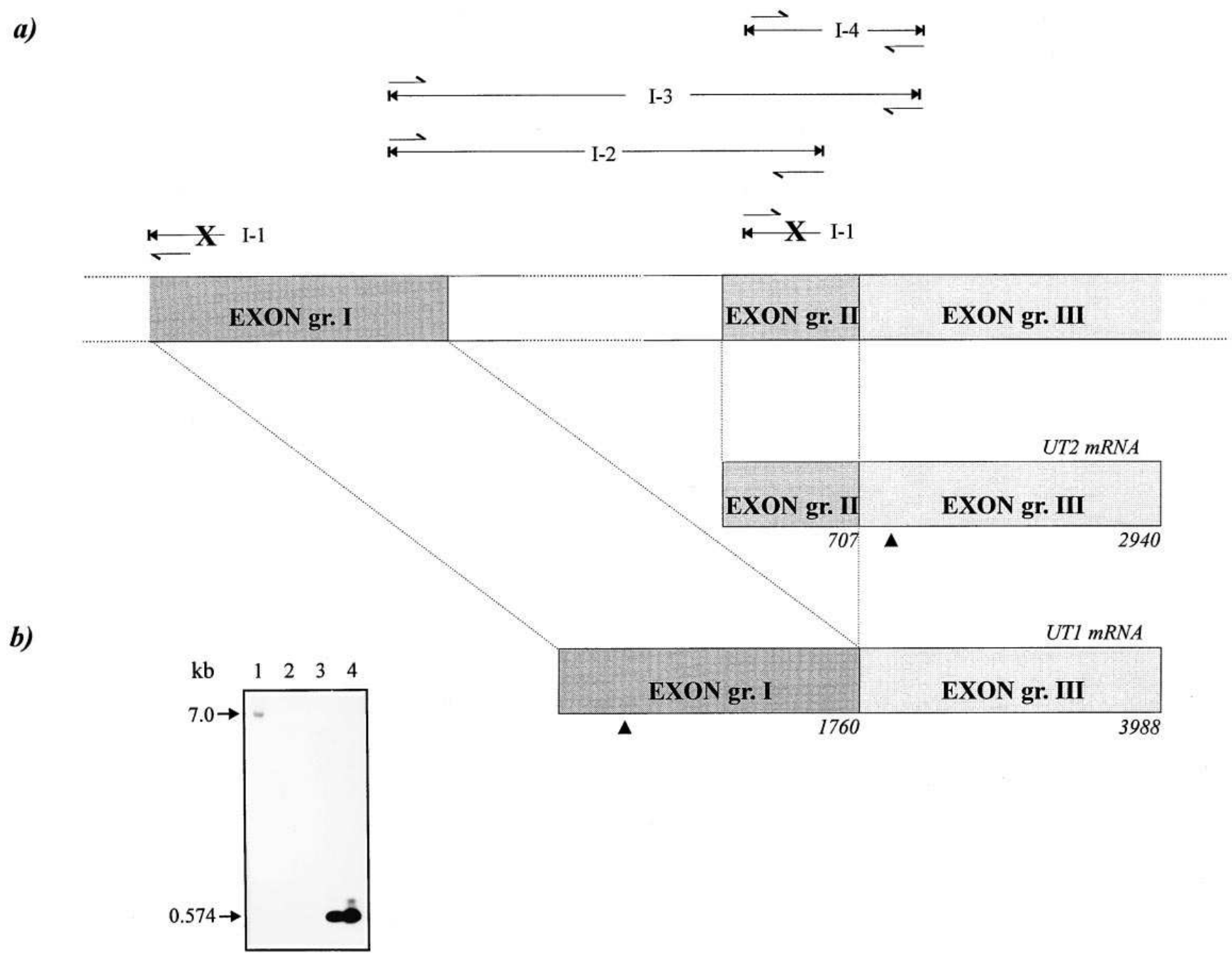

Figure 6. Schematic representation of the rat UT gene showing alternative splicing of UT1 and UT2 cDNA. (a) The positions of exon groups I and II (dark shading) within the UT1 and UT2 mRNAs are indicated by the dashed lines. Exon group III (light shading) is common to both UT1 and UT2. The arrowheads represent the initiation codons of the UT1 and UT2 open reading frames and the nucleotide positions of the intron/ exon boundaries are indicated. The splicing pattern was identified by the PCR primer sets I-1, I-2, I-3, and I-4 as shown. (b) Southern analysis of PCR products. The PCR products were electrophoresed in an agarose gel and transferred to a nitrocellulose membrane. The filter was probed with a UT2-specific cDNA (corresponding to exon group II) at $42^{\circ} \mathrm{C}$ and washed with $0.1 \times \mathrm{SSC}, 0.1 \% \mathrm{SDS}$ at $65^{\circ} \mathrm{C}$. PCR of genomic DNA with primer set I-2 yielded a $7.0-\mathrm{kb}$ fragment (lane 1) but no product was obtained using UT1 cDNA as a template (lane 2). The I-4 primers produced a fragment of $0.5 \mathrm{~kb}$ from both genomic DNA (lane 3) and UT1 cDNA (lane 4).

cAMP response element which is activated in response to vasopressin in a tissue-specific manner.

In summary, we have isolated and characterized a novel phloretin-sensitive, vasopressin-regulated, urea transporter (UT1) cDNA that is expressed in the inner medullary collecting duct, predominantly in the terminal part. UT1 is the only member of the urea transporter family that is found to be stimulated by cAMP. We have demonstrated that UT1 and UT2 are derived from a single gene through the use of different $5^{\prime}$ splice sites. It is likely that the choice of alternative utilization of splice sites is regulated in a tissue-specific manner by the use of either different promoters upstream of the $5^{\prime}$ termini or different transacting factors, thereby producing UT1 and UT2 mRNA levels that are distinct both in the terms of tissue distribution and responsive stimuli.

\section{Acknowledgments}

The authors thank Craig P. Smith for providing background information on this project.

This work was supported by National Institutes of Health grant R01-DK707844 to M.A. Hediger. C. Shayakul is supported by a research fellowship grant from Siriraj-China Medical Board, Mahidol University, Thailand.

\section{References}

1. Cohen, P.P. 1976. Evolutionary and comparative aspects of urea biosynthesis. John Wiley \& Sons, New York. 21-38.

2. Roy, D.R., H.E. Layton, and R.L. Jamison. 1992. Countercurrent mechanism and its regulation. Raven Press, Ltd., New York. 1649-1692.

3. Knepper, M.A., and F. Roch-Ramel. 1987. Pathways of urea transport in the mammalian kidney. Kidney Int. 31:629-633. 
4. Morgan, T., and R.W. Berliner. 1968. Permeability of the loop of Henle, vasa recta, and collecting duct to water, urea, and sodium. Am. J. Physiol. 215: $108-115$

5. Sands, J.M., H. Nonoguchi, and M.A. Knepper. 1987. Vasopressin effects on urea and $\mathrm{H}_{2} \mathrm{O}$ transport in inner medullary collecting duct subsegments. Am. J. Physiol. 253:F823-F832.

6. Chou, C.L., and M.A. Knepper. 1989. Inhibition of urea transport in inner medullary collecting duct by phloretin and urea analogues. Am. J. Physiol. 257:F359-F365.

7. Star, R.A., and M.A. Knepper. 1990. The vasopressin-regulated urea transporter in renal inner medullary collecting duct. Am. J. Physiol. 259:F393F401.

8. Star, R.A., H. Nonoguchi, R. Balaban, and M.A. Knepper. 1988. Calcium and cyclic adenosine monophosphate as second messengers for vasopressin in the rat inner medullary collecting duct. J. Clin. Invest. 81:1879-1888.

9. Nielsen, S., and M.A. Knepper. 1993. Vasopressin activates collecting duct urea transporters and water channels by distinct physical processes. Am. J. Physiol. 265:F204-F213.

10. You, G., C.P. Smith, Y. Kanai, W.S. Lee, M. Stelzner, and M.A. Hediger. 1993. Cloning and characterization of the vasopressin-regulated urea transporter. Nature (Lond.). 365:844-847.

11. Smith, C.P., W.S. Lee, S. Martial, M.A. Knepper, G. You, J.M. Sands, and M.A. Hediger. 1995. Cloning and regulation of expression of the rat kidney urea transporter (rUT2). J. Clin. Invest. 96:1556-1563.

12. Hasegawa, H., and A.S. Verkman. 1993. Functional expression of
cAMP-dependent and independent urea transporters in Xenopus oocytes. Am. J. Physiol. 265:C514-C520.

13. Wells, R.G., A.M. Pajor, Y. Kanai, E. Turk, E.N. Wright, and M.A. Hediger. 1992. Cloning of a human kidney cDNA with similarity to the sodium glucose transporter. Am. J. Physiol. 263:F459-F465.

14. Kanai, Y., M.G. Stelzner, W.S. Lee, R.G. Wells, D. Brown, and M.A. Hediger. 1992. Expression of mRNA (D2) encoding a protein involved in amino acid transport in S3 proximal tubule. Am. J. Physiol. 263:F1087-F1092.

15. Star, R.A. 1990. Apical membrane limits urea permeation across the rat inner medullary collecting duct. J. Clin. Invest. 86:1172-1178.

16. Wall, S.M., J.S. Han, C.L. Chou, and M.A. Knepper. 1992. Kinetics of urea and water permeability activation by vasopressin in rat terminal IMCD. Am. J. Physiol. 262:F989-F998.

17. Knepper, M.A., S. Nielsen, C.L. Chou, and S.R. DiGiovanni. 1994 Mechanism of vasopressin action in the renal collecting duct [Review]. Semin. Nephrol. 14:302-321.

18. Jung, J.S., G.M. Preston, B.L. Smith, W.B. Guggino, and P. Agre. 1994. Molecular structure of the water channel through aquaporin CHIP. The hourglass model. J. Biol. Chem. 269:14648-14654.

19. Olives, B., P. Neau, P. Bailly, M.A. Hediger, G. Rousselet, J.P. Cartron, and P. Ripoche. 1994. Cloning and functional expression of a urea transporter from human bone marrow cells. J. Biol. Chem. 269:31649-31652.

20. Vallejo, M., and J.F. Habener. 1994. Mechanisms of transcriptional regulation by cAMP. In Transcription: Mechanisms and Regulation. R.C. Conaway and J.W. Conaway, editors. Raven Press, Ltd., New York. 353-368. 